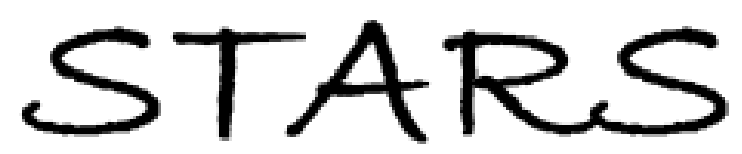

University of Central Florida

STARS

Faculty Bibliography 2010s

Faculty Bibliography

$1-1-2010$

\title{
Wave functions and energy spectra for the hydrogenic atom in R-3 $x M$
}

Robert A. Van Gorder

University of Central Florida

Find similar works at: https://stars.library.ucf.edu/facultybib2010 University of Central Florida Libraries http://library.ucf.edu

This Article is brought to you for free and open access by the Faculty Bibliography at STARS. It has been accepted for inclusion in Faculty Bibliography 2010 s by an authorized administrator of STARS. For more information, please contactSTARS@ucf.edu.

\section{Recommended Citation}

Van Gorder, Robert A., "Wave functions and energy spectra for the hydrogenic atom in R-3 x M" (2010). Faculty Bibliography 2010s. 884.

https://stars.library.ucf.edu/facultybib2010/884

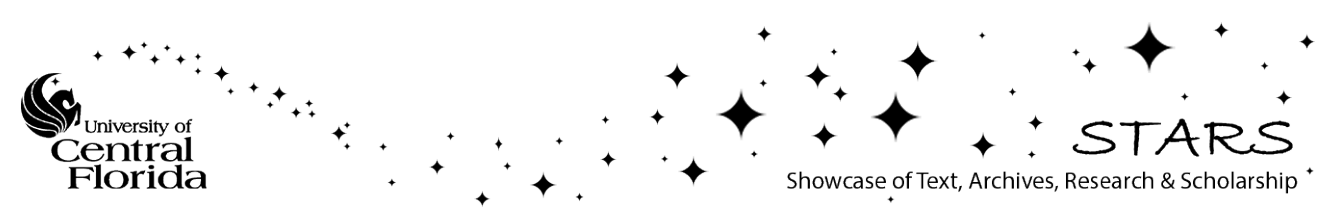




\section{Wave functions and energy spectra for the hydrogenic atom in $\mathbb{R}^{3} \times \mathcal{M}$}

Cite as: J. Math. Phys. 51, 122104 (2010); https://doi.org/10.1063/1.3520507

Submitted: 13 August 2010 . Accepted: 03 November 2010. Published Online: 16 December 2010

Robert A. Van Gorder

ARTICLES YOU MAY BE INTERESTED IN

Schrödinger's equation and general relativity

Journal of Mathematical Physics 41, 5256 (2000); https://doi.org/10.1063/1.533406

Separation of variables on $\mathrm{n}$-dimensional Riemannian manifolds. I. The $\mathrm{n}$-sphere $\mathrm{S}_{\mathrm{n}}$ and Euclidean $n$-space $R_{n}$

Journal of Mathematical Physics 27, 1721 (1986); https://doi.org/10.1063/1.527088

Motion of isolated open vortex filaments evolving under the truncated local induction approximation

Physics of Fluids 29, 115105 (2017); https://doi.org/10.1063/1.5005113

Where in the world is AIP Publishing? Find out where we are exhibiting next 


\title{
Wave functions and energy spectra for the hydrogenic atom in $\mathbb{R}^{3} \times \mathcal{M}$
}

\author{
Robert A. Van Gordera) \\ Department of Mathematics, University of Central Florida, Orlando, Florida 32816, USA
}

(Received 13 August 2010; accepted 3 November 2010; published online 16 December 2010)

\begin{abstract}
We consider the hydrogenic atom in a space of the form $\mathbb{R}^{3} \times \mathcal{M}$, where $\mathcal{M}$ may be a generalized manifold obeying certain properties. We separate the solution to the governing time-independent Schrödinger equation into a component over $\mathbb{R}^{3}$ and a component over $\mathcal{M}$. Upon obtaining a solution to the relevant eigenvalue problems, we recover both the wave functions and energy spectrum for the hydrogenic atom over $\mathbb{R}^{3} \times \mathcal{M}$. We consider some specific examples of $\mathcal{M}$, including the fairly simple $D$-dimensional torus $T^{D}$ and the more complicated Kähler conifold $\mathcal{K}$ in order to illustrate the method. In the examples considered, we see that the corrections to the standard energy spectrum for the hydrogen atom due to the addition of higher dimensions scale as a constant times $1 / L^{2}$, where $L$ denotes the size of the additional dimensions. Thus, under the assumption of small compact extra dimensions, even the first energy corrections to the standard spectrum will be quite large. () 2010 American Institute of Physics. [doi:10.1063/1.3520507]
\end{abstract}

\section{INTRODUCTION}

Since the time that Gunnar Nordström introduced an extra spatial dimension into his theory of gravitation in 1914, ${ }^{1}$ simultaneously describing gravity and electromagnetism (albeit, incorrectly) while also serving as an instigator of the Kaluza-Klein theory, ${ }^{2}$ scientists have postulated the need for, and, influence of, additional spatial dimensions in models of the physical world. Indeed, since its inception as a dual resonance model describing strongly interacting hadrons as "strings," string theory in particular serves as an example of one field in theoretical physics for which the application of additional dimensions has been fruitful. While there has been a spate of recent activity concerning extra dimensions, relatively little literature exists regarding atoms in higher spatial dimensions than three, particularly in the case in which the extra dimensions are compact; such is the motivation of the present work. We should mention that some results for the higher-dimensional Kepler problem do exist; see, e.g., Mladenov and Tsanov. ${ }^{3}$

In the present paper, we shall consider a hydrogenic atom over a space of the form $\mathbb{R}^{3} \times \mathcal{M}$, where $\mathcal{M}$ is taken to be (in the most general setting) a generalized manifold. We solve the timeindependent Schrödinger equation over such a space via separation of variables, which results in two eigenvalue problems, one corresponding to $\mathbb{R}^{3}$ and one corresponding to $\mathcal{M}$. Assuming that such a solution exists to the eigenvalue problem over $\mathcal{M}$, in Sec. II we present a general method by which one may obtain the wave function and energy spectrum for a hydrogenic atom over $\mathbb{R}^{3} \times \mathcal{M}$. Then, in Sec. III, we apply these methods to obtain the wave functions and energy spectrum for the hydrogenic atom over $\mathbb{R}^{3} \times \mathcal{M}$, for specific examples of $\mathcal{M}$. In particular, we consider the $D$-dimensional torus $T^{D}$ as an example of a fairly simple manifold, along with a Kähler conifold $\mathcal{K}$ which serves as an example of a more complicated space. In the examples corresponding to compact extra dimensions, we see that the corrections to the standard energy spectrum for the hydrogen atom due to the higher dimensions scales as $1 / L^{2}$, where $L$ denotes the size of the additional dimensions, and we comment on this more in Sec. IV, where we apply a general result from the analysis on

\footnotetext{
${ }^{a)}$ Electronic mail: rav@ knights.ucf.edu.
} 
manifolds to arrive at a lower bound on the magnitude of the energy corrections due to the compact extra dimensions. It is clear, then, that under the assumption of "small" compact extra dimensions, even the first energy corrections to the standard spectrum will be quite large. Indeed, if there are compact extra dimensions, they must have been sufficiently small to have escaped experimental verification thus far. In obtaining the results of Secs. II-III, we needed to make an assumption on the coulomb potential so as to apply separation of variables, and in Sec. V we discuss how reasonable this assumption may be. Finally, in Sec. VI, we discuss the extension of the method to more complicated atoms, giving the Helium atom over $\mathbb{R}^{3} \times \mathcal{M}$ as an example. Computational difficulties and simplifying approximations are discussed.

\section{GENERAL SOLUTION METHOD FOR THE HYDROGENIC ATOM IN $\mathbb{R}^{3} \times \mathcal{M}$}

We set out to obtain some fairly general expressions for the wave function and energy spectrum governing a hydrogenic atom over the space $\mathbb{R}^{3} \times \mathcal{M}$. In order to find the wave function $\Psi$ and associated energy spectrum $E$ for the hydrogenic atom in higher dimensions than three, we consider the eigenvalue problem (a time-independent Schrödinger equation)

$$
\hat{\mathcal{H}} \Psi=E \Psi, \text { where } \hat{\mathcal{H}}=-\frac{\hbar^{2}}{2 \mu} \Delta+U,
$$

over $\mathbb{R}^{3} \times \mathcal{M}$, where $\hat{\mathcal{H}}$ is an elliptic operator, $\triangle$ is the Laplacian over $\mathbb{R}^{3} \times \mathcal{M}, U$ is the potential energy (we will, in general, assume a potential $U=U(r)$ for mathematical simplicity), and $\mathcal{M}$ is a generalized manifold over which we may perform separation of variables so that $\triangle=\triangle_{\mathbb{R}^{3}}+\triangle_{\mathcal{M}}$ with $\triangle_{\mathbb{R}^{3}}$ and $\triangle_{\mathcal{M}}$ denoting Laplacians over $\mathbb{R}^{3}$ and $\mathcal{M}$, respectively. (More accurately, $\triangle_{\mathcal{M}}$ denotes the Laplace-Beltrami operator over $\mathcal{M}$.) Then, assuming a separable solution to (2.1) of the form $\Psi=\Psi_{1} \Psi_{2}$, where $\Psi_{1}: \mathbb{R}^{3} \rightarrow \mathbb{C}$ and $\Psi_{2}: \mathcal{M} \rightarrow \mathbb{C}$, we have that $\triangle \Psi=\Psi_{2} \triangle_{\mathbb{R}^{3}} \Psi_{1}+\Psi_{1} \triangle_{\mathcal{M}} \Psi_{2}$, which allows us to write (2.1) as a system of coupled linear eigenvalue problems, to wit:

$$
\begin{gathered}
-\frac{\hbar^{2}}{2 \mu} \triangle_{\mathbb{R}^{3}} \Psi_{1}+\left(U-E-\Gamma_{\mathcal{M}}\right) \Psi_{1}=0, \\
-\frac{\hbar^{2}}{2 \mu} \triangle_{\mathcal{M}} \Psi_{2}+\Gamma_{\mathcal{M}} \Psi_{2}=0,
\end{gathered}
$$

where the spectrum $\Gamma_{\mathcal{M}}$ for $\Psi_{2}$ is obtained by the solution of the latter problem (2.3) over $\mathcal{M}$ and then placed into (2.2) in order to obtain $\Psi_{1}$ and hence the full wave function, $\Psi$. In principle, $\Gamma_{\mathcal{M}}$ provides us with the corrections to the standard energy spectra, $E_{N}$ (for a hydrogen atom in $\mathbb{R}^{3}$ ), that are needed due to the assumption of extra dimensions which enter due to $\mathcal{M}$. Naturally, the spectrum $\Gamma_{\mathcal{M}}$ will depend strongly on the choice of $\mathcal{M}$. For now, we shall keep $\mathcal{M}$ arbitrary and assume that a nontrivial solution $\Psi_{2}$ to the eigenvalue problem (2.3) exists and admits nontrivial spectrum $\left\{\left(\Gamma_{\mathcal{M}}\right)_{k}\right\}_{k \in K}$ (where $K$ is an index set). We then solve (2.2) subject to such an assumption and obtain both the wave function and energy spectrum for a hydrogenic atom, in terms of $\Psi_{2}$ and the spectrum $\Gamma_{\mathcal{M}}$. Later, in Sec. III, we fix $\mathcal{M}$ so that we may obtain explicit expressions for the wave function $\Psi_{2}$ and spectrum $\Gamma_{\mathcal{M}}$ in some interesting cases.

Under such assumptions, (2.2) becomes

$$
-\frac{\hbar^{2}}{2 \mu} \triangle_{\mathbb{R}^{3}} \Psi_{1}+\left(U-E-\left(\Gamma_{\mathcal{M}}\right)_{k}\right) \Psi_{1}=0 .
$$

Assuming that $\Psi_{1}$ separates as $\left(\Psi_{1}\right)_{l}(r, \theta, \phi)=R(r) Y_{l}(\theta, \phi)$, where $R(r)$ is the radial component and $Y_{l}(\theta, \phi)$ is a spherical harmonic (here, the index $\ell$ runs as $\ell \in \mathbb{N}$ ). Taking the Laplacian $\triangle_{\mathbb{R}^{3}}$ to spherical coordinates, $(2.4)$ results in the coupled eigenvalue problems

$$
-\frac{\hbar^{2}}{2 \mu}\left(\frac{1}{R} \frac{d}{d r}\left(r^{2} \frac{d R}{d r}\right)\right)+r^{2}\left(U-E-\left(\Gamma_{\mathcal{M}}\right)_{k}\right)=-\frac{\hbar^{2}}{2 \mu} \lambda,
$$




$$
\frac{\hbar^{2}}{2 \mu}\left(\frac{1}{Y \sin \theta} \frac{\partial}{\partial \theta}\left(\sin \theta \frac{\partial Y}{\partial \theta}\right)+\frac{1}{Y \sin ^{2} \theta} \frac{\partial^{2} Y}{\partial \phi^{2}}\right)=-\frac{\hbar^{2}}{2 \mu} \lambda,
$$

where $\lambda$ is a constant. It is well known that a solution $Y_{\ell}(\theta, \phi)$ to (2.6) results in eigenvalues $\lambda_{\ell}=\ell(\ell+1)$ (the square of the angular momentum) where $\ell \in \mathbb{N}$. Then, placing this expression into (2.5), we obtain the radial equation

$$
-\frac{\hbar^{2}}{2 \mu}\left(\frac{1}{R} \frac{d}{d r}\left(r^{2} \frac{d R}{d r}\right)-\ell(\ell+1)\right)+r^{2}\left(U-E-\left(\Gamma_{\mathcal{M}}\right)_{k}\right)=0 .
$$

Setting $U$ to the Coulomb potential (that is, $\left.U=U(r)=-Z e^{2} / r\right)$, defining constants $a_{\ell}=\ell(\ell+$ 1), $b=-2 \mu Z e^{2} / \hbar^{2}, c_{k}=-\left(2 \mu / \hbar^{2}\right)\left(E+\left(\Gamma_{\mathcal{M}}\right)_{k}\right)$, and making the transformation of dependent variable $R(r)=r^{-1} S(r)$, the radial equation (2.7) becomes

$$
\frac{d^{2} S}{d r^{2}}-\left(\frac{a_{\ell}+b r+c_{k} r^{2}}{r^{2}}\right) S=0 .
$$

The general solution $S(r)$ to (2.8) may be given in terms of Whittaker M and W functions ${ }^{4}$ as

$$
\begin{aligned}
S(r)= & C_{1} \mathrm{M}\left(-\frac{b}{2 \sqrt{c_{k}}}, \frac{1}{2} \sqrt{1+4 a_{\ell}}, 2 \sqrt{c_{k}} r\right) \\
& +C_{2} \mathrm{~W}\left(-\frac{b}{2 \sqrt{c_{k}}}, \frac{1}{2} \sqrt{1+4 a_{\ell}}, 2 \sqrt{c_{k}} r\right),
\end{aligned}
$$

where $C_{1}$ and $C_{2}$ are constants of integration. As we desire only those solutions that are regular at $r=0$, we take $C_{2}=0$. Putting $S(r)$ in terms of the generalized Laguerre polynomials, we have that

$$
\Psi_{\ell, k}(r, \theta, \phi, \mathbf{w})=\frac{(-1)^{2 \ell+1}\left(2 \sqrt{c_{k}} r\right)^{\ell} e^{-\sqrt{c_{k}} r}}{q !\left(\begin{array}{c}
q \\
2 \ell+1
\end{array}\right)} L_{q}^{2 \ell+1}\left(2 \sqrt{c_{k}} r\right) Y_{\ell}(\theta, \phi) \Psi_{2, k}(\mathbf{w}),
$$

where $\mathbf{w} \in \mathcal{M}$ and $q=\ell-\frac{b}{2 \sqrt{c_{k}}}$. Now, as we require $\Psi_{1} \in L^{2}\left(\mathbb{R}^{3}\right)$, the quantization condition $\ell+1+\frac{b}{2 \sqrt{c_{k}}}=-m_{r}$, where $m_{r} \in \mathbb{N}$ is referred to as the radial quantum number, must hold (and hence $q=2 \ell+1+m_{r} \in \mathbb{N}^{*}$ ). Then, defining $N=m_{r}+\ell$, we have that the condition $-\left(2 \mu / \hbar^{2}\right)\left(E+\left(\Gamma_{\mathcal{M}}\right)_{k}\right)=c_{k}=\frac{b^{2}}{4(N+1)^{2}}$ holds and, in fact, yields the "closed-form" expression for the energy spectrum for the hydrogenic atom over $\mathbb{R}^{3} \times \mathcal{M}$, viz.,

$$
E=E_{N, k}=-\frac{\mu\left(Z e^{2}\right)^{2}}{2 \hbar^{2}(N+1)^{2}}-\left(\Gamma_{\mathcal{M}}\right)_{k}
$$

Clearly, for $\left(\Gamma_{\mathcal{M}}\right)_{0}=0, E_{N, 0}$ from (2.11) gives the standard energy spectrum for the hydrogen atom over $\mathbb{R}^{3}$. Thus, the term $\left(\Gamma_{\mathcal{M}}\right)_{k}$ appears as a "correction" to the standard energy spectrum, to account for the additional dimensions inherent in the model due to the assumption of an underlying space of the form $\mathbb{R}^{3} \times \mathcal{M}$. For precision, note that the indices in the above formulas run as $\ell \in \mathbb{N}, m_{r} \in \mathbb{N}$ and hence $N \in \mathbb{N}$.

\section{EXAMPLES OF WAVE FUNCTIONS AND ENERGY SPECTRA FOR SOME SPECIFIC $\mathcal{M}$}

In order to better illustrate the physical relevance of the results contained in the previous section, we consider both examples and a nonexample of possible spaces $\mathbb{R}^{3} \times \mathcal{M}$ over which one may wish to consider a hydrogenic atom. After presenting the various physical difficulties inherent in assuming that the underlying space might take the form $\mathbb{R}^{D}$ for $D \geq 4$ (and hence that the extra dimensions, if any, are "large"), we present two cases in which the extra dimensions $\mathcal{M}$ may be "small". The first of these cases, the $D$-dimensional torus $T^{D}$, is provided as a simple compact manifold over which we may perform the computations outlined in the general method of the preceeding section. We then consider a more complicated space by assuming that the extra dimensions are due to a Kähler conifold $\mathcal{K}$. In each of these examples, we find that the corrections to the standard energy spectrum 
for the hydrogen atom due to the assumption of additional dimensions scale as the inverse of the square of the size of the extra compact dimensions. In particular, even the first nontrivial level for such corrections is found to be quite large (the lower bound on such a correction is on the order of $10^{18} \mathrm{eV}$; in reality this might even be much larger), which might explain why the influence of compact extra dimensions have eluded experimental verification.

\section{A. The case of $\mathbb{R}^{D}, D \geq 4$}

In the literature, several authors ${ }^{5}$ have considered a hydrogenic atom on $\mathbb{R}^{D}$ for $D \geq 4$, and even further generalizations to other atoms ${ }^{6}$ have taken place over $\mathbb{R}^{D}$. Typically, the wave function is assumed to take the form $\Phi=R(r) Y\left(\theta_{1}, \theta_{2}, \ldots, \theta_{D-1}\right)$, where $Y$ is a hyperspherical harmonic and $R(r)$ is the radial function. Under such an assumption, the radius $r$ is free to run over all of $\mathbb{R}^{D}$, as opposed to only $\mathbb{R}^{3}$; that is, $r$ behaves as a hyperradius. As such, this should necessitate a change in Maxwell's equations to account for the added $D-3$ "large" dimensions over which $r$ may span. As a result, some authors have considered the modified Coulomb potential $U(r)=-Z e^{2} / r^{D-2}$ for $D \geq 3$ and $U(r)=Z e^{2} \log r$ for $D=2$ (see, for instance, Refs. 7 and 8). Assuming such a potential, the wave equation and energy spectrum for the hydrogenic atom over $\mathbb{R}^{2}$ has been determined. ${ }^{8}$ Unfortunately, for $D \geq 4$, such an assumption on the potential $U(r)$ results in problems. ${ }^{9}$ For $D=4$, the assumption of such a potential results in an overdetermined system for the energy spectrum which admits no nontrivial solutions. Furthermore, in the case of $D \geq 5$, the assumption of a potential of this form results in a radial equation with an irregular singular point at $r=0$. Physically, such a potential results in unstable orbits for such a hydrogenic atom whenever $D \geq 5$.

With such difficulties in mind, most authors to consider a hydrogenic atom over $\mathbb{R}^{D}$ when $D \geq 4$ keep the standard Coulomb potential $U(r)=-Z e^{2} / r$ (Ref. 10), and obtain a wave function of the form

$$
\Psi\left(r, \theta_{1}, \theta_{2}, \ldots, \theta_{D-1}\right)=C_{N, \ell}(2 \alpha r)^{\ell} e_{1}^{-\alpha r} F_{1}\left(-n_{r}, 2 \ell+D-1,2 \alpha r\right) Y_{\ell}\left(\theta_{1}, \theta_{2}, \ldots, \theta_{D-1}\right),
$$

where $\alpha=\sqrt{-2 \mu E} / \hbar,-n_{r}=\ell+\frac{1}{2}(D-1)-\left(\mu Z e^{2}\right) /\left(\alpha \hbar^{2}\right), \ell \in \mathbb{N}, n_{r} \in \mathbb{N}, N=n_{r}+\ell \in \mathbb{N}$, ${ }_{1} F_{1}$ denotes Kummer's confluent hypergeometric function (of the first kind), and $C_{N, \ell}$ is a constant of normalization defined by

$$
C_{N, \ell}=\frac{1}{(2 \ell+D-2) !} \frac{(2 \alpha)^{D}(N+\ell+D-2) !}{(2 N+D-1)(N-\ell) !} .
$$

The energy spectrum is then given by

$$
E_{N}=\frac{E_{0}}{\left(N+\frac{1}{2}(D-1)\right)^{2}}, \quad \text { where } \quad E_{0}=-\frac{\mu\left(Z e^{2}\right)^{2}}{2 \hbar^{2}} .
$$

Note that a shift in the energy spectrum due to the assumption of an underlying space of the form $\mathbb{R}^{D}, D \geq 4$, is clearly incompatable with experimental observations; if such "large" extra dimensions of the form $\mathbb{R}^{D-3}$ were present, they would have been noticed long ago! With this in mind, we should seek models of the hydrogenic atom in which the underlying space has fairly small extra dimensions.

\section{B. The case of $\mathcal{M}=T^{D}$}

We now consider the hydrogenic atom over $\mathbb{R}^{3} \times T^{D}$ for $D \geq 1$, where $T^{D}$ denotes the $D$ dimensional torus. The choice of a $D$-dimensional torus is one of the simpler possibilities for $\mathcal{M}$, and will thus make for an illustrative example.

Let $\mathbf{w} \in T^{D}$. Then, $\Psi_{2}=\Psi(\mathbf{w})$ is the component of the wave function to be solved over the torus, and in particular $\Psi_{2}$ satisfies (2.3) where we denote $\Gamma_{\mathcal{M}}=\Gamma_{T^{D}}$. Now, $T^{D}=S^{1} \times S^{1} \times \cdots \times S^{1}$, the Cartesian product of $D$ 1-spheres (circles) $S^{1}$. To each of these copies of $\mathcal{S}^{1}$, we associate a component $w_{j}, 1 \leq j \leq D$, of the vector $\mathbf{w}$ as well as an arclength $L_{j}, 1 \leq j \leq D$. Without loss of generality, $L_{1} \leq L_{2} \leq \cdots \leq L_{D}$. Defining the constant vector $\mathbf{L}=\left(L_{1}, L_{2}, \ldots, L_{D}\right)$, it is clear that 
$\Psi_{2}(\mathbf{w})$ must satisfy the condition $\Psi_{2}(\mathbf{0})=\Psi_{2}(\mathbf{L})$ on $T^{D}$, where $\mathbf{0}$ denotes the zero vector. With such a condition in mind, we assume a solution to (2.3) of the form

$$
\Psi_{2, \mathbf{n}}(\mathbf{w})=C_{\mathbf{n}} \exp \left(-2 \pi i \sum_{j=1}^{D} \frac{n_{j} w_{j}}{L_{j}}\right),
$$

where $\mathbf{n}=\left(n_{1}, n_{2}, \ldots, n_{D}\right) \in \mathbb{N}^{D}$ is an index vector. Placing this expression back into (2.3), we obtain the spectrum

$$
\left(\Gamma_{\mathbb{T}^{D}}\right)_{\mathbf{n}}=\frac{2 \pi \hbar^{2}}{\mu} \sum_{j=1}^{D}\left(\frac{n_{j}}{L_{j}}\right)^{2} .
$$

Upon setting $\Psi_{2}=\Psi_{2, \mathbf{n}}(\mathbf{w})$ from (3.3) and $\Gamma_{\mathcal{M}}=\Gamma_{T^{D}}$ from (3.4) in (2.10), we have the wave function for the hydrogenic atom over $\mathbb{R}^{3} \times T^{D}$. The energy spectrum for this wave function follows from (2.11) and (3.4), and is given by

$$
E=E_{N, \mathbf{n}}=-\frac{\mu\left(Z e^{2}\right)^{2}}{2 \hbar^{2}(N+1)^{2}}-\frac{2 \pi \hbar^{2}}{\mu} \sum_{j=1}^{D}\left(\frac{n_{j}}{L_{j}}\right)^{2} .
$$

Converting this expression to standard units, we have that

$$
E_{N, \mathbf{n}}=-\frac{13.6 \mathrm{eV}}{(N+1)^{2}}-\left(1.514 \times 10^{-18} \mathrm{eV} \cdot \mathrm{m}^{2}\right) \sum_{j=1}^{D}\left(\frac{n_{j}}{L_{j}}\right)^{2} .
$$

The mode corresponding to the lowest magnitude first correction due to additional dimensions is given by

$$
E_{N,(0, \ldots, 0,1)}=-\frac{13.6 \mathrm{eV}}{(N+1)^{2}}-\frac{1.514 \times 10^{-18} \mathrm{eV} \cdot \mathrm{m}^{2}}{L_{D}^{2}} .
$$

In order to be in agreement with experimentation any additional spatial dimensions should be "small". Assuming a relatively "large" value of $L_{D}$, say on the order of that which might be detected by the large hadron collider (LHC) of CERN, say $L_{D}=10^{-18} \mathrm{~m}^{2}$, we have that the first correction to the standard energy spectrum for the hydrogen atom is of the order of magnitude $10^{18} \mathrm{eV}$. We note that this is a low estimate for the first correction.

\section{The case of $\mathcal{M}=\mathcal{K}$, a Kähler conifold}

As our final specific example, we take $\mathcal{M}=\mathcal{K}$, where $\mathcal{K}$ is a Kähler conifold, ${ }^{12}$ to demonstrate the method for a more complicated space which has found much application in mathematical physics. ${ }^{13}$ Likely one of the most famous examples of a Kähler manifold are the Calabi-Yau manifolds, which are sometimes defined as compact Kähler manifolds whose canonical bundle is trivial (see, e.g., Ref. 14), although an abundance of equivalent definitions exist. We find that many of the statements about the magnitude of the corrections to the energy spectrum made above in the case of the hydrogenic atom over $\mathbb{R}^{3} \times \mathbb{T}^{D}$ translate over to this more complicated case quite nicely. We remark that Refs. 15 and 16 consider the quantum oscillator and Coulomb systems over Kähler conifolds $\mathcal{K}$ entirely (see also Ref. 17), while our results correspond to the hydrogenic atom over $\mathbb{R}^{3} \times \mathcal{K}$, a cartesian product of a Kähler conifold with $\mathbb{R}^{3}$. The results below thus employ the results in Ref. 15, so we shall freely skip steps in the derivation, and the initiated reader is referred to Ref. 15 for more complete derivations. In particular, the existence of a solution to the wave equation (2.3) given a space $\mathcal{M}=\mathcal{K}$ (a Kähler conifold) follows from the existence of a solution to problem outlined in Eqs. (4)-(5) of Ref. 15.

We shall consider the $(\nu, \epsilon)$ parameteric family for four-dimensional Kähler conifolds $\mathcal{K}$; nonsingular special cases include the complex projective space $\mathbb{C} \mathrm{P}^{2}$ (when $v=\epsilon=1$ ) and its noncompact counterpart, the four-dimensional Lobacewski space $\mathcal{L}_{2}$ (when $v=-\epsilon=1$ ). In general, the Kähler 
structure is defined by the potential $Q=\frac{r_{0}^{2}}{2 \epsilon} \log \left(1+\epsilon(w \bar{w})^{\nu}\right)$ for $\nu>0$ and $\epsilon= \pm 1$, so that the corresponding metric on $\mathcal{K}$ is given by

$$
g_{a \bar{b}}=\frac{v r_{0}^{2}(w \bar{w})^{\nu-1}}{2\left(1+\epsilon(w \bar{w})^{\nu}\right)}\left(\delta_{a \bar{b}}-\frac{1+v+\epsilon(w \bar{w})^{\nu}}{w \bar{w}\left(1+\epsilon(w \bar{w})^{\nu}\right)} \bar{w}^{a} w^{b}\right),
$$

while the scalar curvature is given by

$$
\kappa=-\frac{4}{v r_{0}^{2}}\left(\frac{v-1-\epsilon(2 v+1)(w \bar{w})^{v}}{w \bar{w}}\right) .
$$

Then, the Laplacian in (2.3) becomes $\Delta_{\mathcal{K}}=g^{a \bar{b}} \partial_{a} \partial_{\bar{b}}$, where $\partial_{a}=\partial / \partial w^{a}$ and $\partial_{\bar{a}}=\partial / \partial w^{\bar{a}}$. As mentioned in Ref. 15, such an equation (2.3) with the given Laplacian is exactly solvable over $\mathcal{K}$. That is to say, one may obtain the spectrum $\left(\Gamma_{\mathcal{K}}\right)_{k}$ for (2.3) and then construct a complete basis of wave functions for the problem.

Taking $\mathbf{w} \in \mathcal{K}$ into the coordinates $\mathbf{w}=\left(x, \theta_{1}, \theta_{2}, \theta_{3}\right)$, where $\theta_{1} \in[0,2 \pi), \theta_{2} \in[0, \pi], \theta_{3} \in$ $[0,4 \pi)$, and either $x \in[0, \infty)$ when $\epsilon=1$ or $x \in[0,1]$ when $\epsilon=-1,{ }^{15}$ assume a wave function $\Psi_{2}: \mathcal{K} \rightarrow \mathbb{R}$ of the form

$$
\Psi_{2}(\mathbf{w})=\rho(x) \mathrm{D}_{m, s}^{j}\left(\theta_{1}, \theta_{2}, \theta_{3}\right),
$$

where $\mathrm{D}_{m, s}^{j}$ denotes the Wigner function, ${ }^{18,19} J$ and $m$ denote the orbital and azimuthal quantum numbers, respectively, and $s$ is the eigenvalue of the operator $\hat{J}_{0} \Psi_{2}=s \Psi_{2}, \hat{\mathbf{J}}^{2} \Psi_{2}=j(j+1) \Psi_{2}$, $\hat{J}_{3} \Psi_{2}=m \Psi_{2}$, where $m, s=-j,-j+1, \ldots, j-1, j$ for $j=0, \frac{1}{2}, 1, \ldots$. The volume element is then

$$
d V_{(4)}=\frac{v^{2} r_{0}^{4}}{32} \frac{x^{3}}{\left(1+x^{2}\right)^{3}} \sin \theta_{2} d x d \theta_{1} d \theta_{2} d \theta_{3} .
$$

Then, placing (3.8) into (2.3), we find that $\rho(x)$ is the solution to the radial equation

$$
\frac{d^{2} \rho(x)}{d x^{2}}+\frac{3+\epsilon x^{2}}{1+\epsilon x^{2}} \frac{1}{x} \frac{d \rho(x)}{d x}+\left(\frac{4 \mu r_{0}^{2} E}{\hbar^{2}\left(1+\epsilon x^{2}\right)^{2}}-\frac{4 v j(j+1)+4(1-v) s^{2}}{v^{2} x^{2}\left(1+\epsilon x^{2}\right)}-\frac{\epsilon \delta^{2}}{1+\epsilon x^{2}}\right) \rho(x)=0,
$$

where $\delta=2 s / \nu$. Making the chance of variable $x=\tan \phi$ for $\epsilon=1$ and $x=\tanh \phi$ for $\epsilon=-1$, we obtain the regular radial part of the $\Psi_{2}$ component of the wave function, which reads

$$
\rho(\phi)=C_{n, j, s} \sin ^{j_{1}-1}(\phi) \cos ^{\delta}(\phi){ }_{2} F_{1}\left(-n, n+\delta+j_{1}+1 ; j_{1}+1 ; \sin ^{2}(\phi)\right)
$$

for $\epsilon=1$ and

$$
\rho(\phi)=C_{n, j, s} \sinh ^{j_{1}-1}(\phi) \cosh ^{2 n-\delta}(\phi){ }_{2} F_{1}\left(-n,-n+\delta ; j_{1}+1 ; \tanh ^{2}(\phi)\right)
$$

for $\epsilon=-1$, where ${ }_{2} F_{1}$ denotes Gauss' hypergeometric function, ${ }^{20} j_{1}$ is defined by

$$
j_{1}^{2}=\frac{4 j(j+1)}{v}+1-(v-1) \delta^{2},
$$

and $n$ is the radial number, where $n=0,1, \ldots, \infty$ for $\epsilon=1$ or $n=0,1, \ldots, n{ }^{\max }=[\delta / 2-j-1]$ for $\epsilon=-1$. The normalization constants $C_{n, j, s}$ are defined by the relation

$$
\frac{v^{2} r_{0}^{4} \pi^{2} n ! \Gamma^{2}\left(j_{1}+1\right)}{4(2 j+1) \Gamma\left(n+j_{1}+1\right)} C_{n, j, s}=\Lambda_{n, j, s}(\epsilon)
$$

where

$$
\Lambda_{n, j, s}(1)=\frac{\left(2 n+j_{1}+1+\delta\right) \Gamma\left(n+j_{1}+1+\delta\right)}{\Gamma(n+1+\delta)} \quad \text { and } \quad \Lambda_{n, j, s}(-1)=\frac{\left(\delta-2 n-j_{1}-1\right) \Gamma(\delta-n)}{\Gamma\left(\delta-n-j_{1}\right)} .
$$

We thus have a solution $\Psi_{2}$ for the component of the wave function over the Kähler conifold with parameters $v>0$ and $\epsilon= \pm 1$. Placing this back into (2.10), we obtain the wave function $\Psi_{l, \mathbf{k}}$ for the hydrogenic atom over $\mathbb{R}^{3} \times \mathcal{K}$, where $\mathbf{k}=(n, j, s)$ is an index vector. 
With such a wave function $\Psi_{l, \mathbf{k}}$, the spectrum for (2.3) is found to be

$$
\left(\Gamma_{\mathcal{K}}\right)_{\mathbf{k}}=\frac{\epsilon \hbar^{2}}{4 \mu r_{0}^{2}} \Omega_{\mathbf{k}}, \quad \text { where } \quad \Omega_{\mathbf{k}}=\left(2 n+j_{1}+\epsilon \delta+1\right)^{2}-4
$$

Then, the associated energy spectrum for the hydrogenic atom over $\mathbb{R}^{3} \times \mathcal{K}$ is given by placing (3.12) into (2.11). We obtain

$$
E=E_{N, \mathbf{k}}=-\frac{\mu\left(Z e^{2}\right)^{2}}{2 \hbar^{2}(N+1)^{2}}-\frac{\epsilon \hbar^{2}}{4 \mu r_{0}^{2}} \Omega_{\mathbf{k}}
$$

or, in standard units,

$$
E=E_{N, \mathbf{k}}=-\frac{13.6 \mathrm{eV}}{(N+1)^{2}}-\left(\frac{6.024 \times 10^{-20} \mathrm{eV} \cdot \mathrm{m}^{2}}{r_{0}^{2}}\right) \epsilon \Omega_{\mathbf{k}} .
$$

For sake of example, let us consider the mode corresponding to $\mathbf{k}=(0,0,1)$; that is,

$$
E_{N,(0,0,1)}=-\frac{13.6 \mathrm{eV}}{(N+1)^{2}}-\left(\frac{2.410 \times 10^{-19} \mathrm{eV} \cdot \mathrm{m}^{2}}{r_{0}^{2}}\right) \epsilon\left((1+\epsilon)^{2}-1\right)
$$

As in the case of the torus, let us assume a relatively "large" value for $r_{0}$, say $r_{0}=5.0 \times 10^{-19} \mathrm{~m}$. We then have from (3.15) that the correction for $E_{N,(0,0,1)}$ due to the assumption of extra dimensions of the form $\mathcal{K}$ is of the order $10^{18}$ to $10^{19} \mathrm{eV}$, depending on the choice of $\epsilon= \pm 1$.

Extensions of the present results to higher-dimensional Kähler conifolds are more or less straightforward, with the introduction of additional independent variables in the Wigner function term. For such higher-dimensional cases, the qualitative results (e.g., the form of the wave function and the obtained energy spectrum) would be comparable to the results presented in the case of a four-dimensional Kähler conifold.

One can comment here that the most appropriate candidates in the setting of the paper are manifolds from the Cartan list of symmetric spaces, as their spectra are well understood (cf. Ref. 21).

\section{COMMENTS ON THE FIRST NONTRIVIAL ENERGY STATE DUE TO SMALL EXTRA DIMENSIONS}

As was illustrated by specific examples in the previous two subsections, the first nontrivial correction to the standard energy spectra due to the small extra dimensions is rather large (on the order of $10^{18}$ to $10^{19} \mathrm{eV}$ for the examples considered). For certain manifolds $\mathcal{M}$, more general results are possible.

Let $\lambda_{1}$ denote the first nontrivial eigenvalue of the Laplace equation on a manifold $\mathcal{M}(0=$ $\left.\lambda_{0}<\lambda_{1} \leq \lambda_{2} \leq \cdots\right)$. Li and Yau $^{22}$ proved that if $\mathcal{M}$ is a closed Riemannian manifold whose Ricci curvature is nonnegative, then $\lambda_{1} \geq \frac{\pi}{2(\operatorname{diam}(\mathcal{M}))^{2}}$, where $\operatorname{diam}(\mathcal{M})$ is the diameter of $\mathcal{M}$. (This was achieved via a gradient estimate on the first eigenfunction.) Later, Yang and Zhong ${ }^{23}$ improved $^{2}$ this estimate by showing that (under the same conditions on $\mathcal{M}) \lambda_{1} \geq \frac{\pi}{(\operatorname{diam}(\mathcal{M}))^{2}}$. This in turn was extended by Yang, ${ }^{24}$ who found that if for some constant $\beta \geq 0, \operatorname{Ric}(X, X) \geq(\operatorname{dim}(\mathcal{M})-1) \beta \geq 0$ for all $X \in \mathcal{T}(\mathcal{M})$, then

$$
\lambda_{1} \geq \frac{(\operatorname{dim}(\mathcal{M})-1)}{4} \beta+\frac{\pi}{(\operatorname{diam}(\mathcal{M}))^{2}} .
$$

Translating this result of Yang $^{24}$ back into our notation, we have that

$$
\left(\Gamma_{\mathcal{M}}\right)_{1} \geq \frac{\hbar^{2}}{2 \mu}\left(\frac{(\operatorname{dim}(\mathcal{M})-1)}{4} \beta+\frac{\pi}{(\operatorname{diam}(\mathcal{M}))^{2}}\right) \geq \frac{\pi \hbar^{2}}{2 \mu(\operatorname{diam}(\mathcal{M}))^{2}}
$$


Hence, assuming that $\mathcal{M}$ at least a closed Riemannian manifold whose Ricci curvature is nonnegative, the first nontrivial energy state (of the form 2.11) due to the small extra dimensions satisfies

$$
E_{N, 1} \leq-\frac{\mu\left(Z e^{2}\right)^{2}}{2 \hbar^{2}(N+1)^{2}}-\frac{\pi \hbar^{2}}{2 \mu(\operatorname{diam}(\mathcal{M}))^{2}}
$$

In keeping with the order of magnitude estimates employed in the previous section, let us take $\operatorname{diam}(\mathcal{M}) \sim 10^{-19} \mathrm{~m}$. Then,

$$
E_{N, 1} \leq-10^{19} \mathrm{eV}
$$

Indeed, if the extra dimensions are even smaller, then this magnitude of the first correction will be quite a bit larger (by two orders of magnitude, for each single order of magnitude decrease in the value $\operatorname{diam}(\mathcal{M})$ ). Thus, the first corrections will be at quite a high energy level, of the order of $1 \mathrm{EeV}$ (one extra electron volt; i.e., one quintillion electron volts). This allows for the impact of the extra dimensions to go unnoticed in the typical energy spectrum for the hydrogen atom. It is interesting to note that, due to the Greisen-Zatsepin-Kuzmin limit (GZK limit) (a theoretical upper limit on the energy of cosmic rays from distant sources; see Ref. 11), we may very well be able to account for why we do not observe such large energies in transitions of energy levels between states corresponding to different values of $\mathbf{n} \in \mathbb{N}^{D}$. (Any such correlation between the GZK limit and energy states for higher-dimensional atoms is, of course, speculative.)

\section{COMMENTS ON THE ASSUMPTION OF A POTENTIAL $U=U(r)$}

In the proceeding analysis, we have always assumed (where needed) that the potential in the Schrödinger equation (2.1) $U=U(r)$; i.e., that the potential depends only on the radial coordinate in $\mathbb{R}^{3}$. Such an assumption permits the separation of variables given by (2.2)-(2.3). While this is certainly a crude approximation to reality, the approximation is likely not very bad (assuming that the extra dimensions are sufficiently small). Suppose instead that $U$ depends on the distance $\|\chi\|$ where $\chi \in \mathbb{R}^{3} \times \mathcal{M}$. If $\mathcal{M}$ is a small compact manifold, we may embed it within some $V_{\delta} \in \mathbb{R}^{2 \operatorname{dim}(\mathcal{M})}$ for some $\delta>\operatorname{diam}(\mathcal{M})$, where $\left\|x_{1}-x_{2}\right\|<\delta$ for all $x_{1}, x_{2} \in V_{\delta}$. Thus, within $\mathbb{R}^{3+2 \operatorname{dim}(\mathcal{M})}$, note that (for $x(\chi) \in V_{\delta}$ )

$$
\|\chi\| \leq\|(r, \theta, \phi, x(\chi))\| \leq r+\|x(\chi)\| \leq r+\delta .
$$

Then,

$$
|U(r)-U(|| \chi||)| \leq|U(r)-U(r+\delta)| .
$$

For small $\delta>0$, this upper bound becomes small (assuming well-behaved potential $U$ ). For instance, when we consider the Coulomb potential $U=U(r)=-Z e^{2} / r$, we find that

$$
|U(r)-U(\|\chi\|)| \leq Z e^{2} \frac{\delta}{(r+\delta) r},
$$

which, for finite $r>0$, tends to zero as $\delta \rightarrow 0$. Hence, for small extra dimension, we may pick $\delta>0$ so that the difference between $U(r)$ and $U(\|\chi\|)$ is negligible. Thus, the assumption $U=U(r)$ does not effect the qualitative results obtained.

\section{EXTENSION OF THE RESULTS TO MORE COMPLICATED ATOMIC STRUCTURES}

Exactly solving the coupled Schrödinger equations is not (in general) possible for the more complicated atoms (as such problems are really "many-body" problems), even for atoms over $\mathbb{R}^{3}$, so we often resort to numerical methods. That said, in the case of simpler atoms, such as the Helium atom, perturbation techniques can be used to obtain approximate solutions. For such atoms, the results are qualitatively similar to those for the hydrogenic atom presented in the previous sections, provided we assume $U(\|x\|) \approx U(r)$. We now demonstrate this with the Helium-like atom over $\mathbb{R}^{3} \times \mathcal{M}$. 
Let $x_{1}, x_{2} \in \mathbb{R}^{3} \times \mathcal{M}$ be distinct, and write $x_{1}=\left(r_{1}, \theta_{1}, \phi_{1}, w_{1}\right), x_{2}=\left(r_{2}, \theta_{2}, \phi_{2}, w_{2}\right)$, where $w_{1}, w_{2} \in \mathcal{M}$. The Schrödinger equation for the Helium-like atom is

$$
\left(H_{1}+H_{2}+\frac{e^{2}}{x_{12}}\right) \Psi=E \Psi
$$

where

$$
H_{i}=-\frac{\hbar^{2}}{2 \mu} \triangle_{x_{i}}-U\left(\left\|x_{i}\right\|\right), \quad x_{12}=\left\|x_{1}-x_{2}\right\| .
$$

Here $\triangle$ is the Laplacian over $\mathbb{R}^{3} \times \mathcal{M}, U$ is the potential energy (we will, in general, assume a potential $U=U\left(\| x_{i}||\right) \approx U(r)$ ), and $\mathcal{M}$ is a generalized manifold over which we may perform separation of variables so that $\triangle=\triangle_{\mathbb{R}^{3}}+\triangle_{\mathcal{M}}$ with $\triangle_{\mathbb{R}^{3}}$ and $\triangle_{\mathcal{M}}$ denoting Laplacians over $\mathbb{R}^{3}$ and $\mathcal{M}$, respectively. For small extra dimensions, we may always find $\delta>0$ such that $D(\mathcal{M})<\delta$. Then, for small $\delta>0$, we have

$$
\frac{1}{x_{12}} \approx \frac{1}{\left|r_{1}-r_{2}\right|}
$$

Thus, in the approximation we consider the coupling will be in the radial variable in $\mathbb{R}^{3}$, alone. Let us assume a wave function of the form

$$
\Psi\left(x_{1}, x_{2}\right)=R\left(r_{1}, r_{2}\right) Y^{[1]}\left(\theta_{1}, \phi_{1}\right) Y^{[2]}\left(\theta_{2}, \phi_{2}\right) W^{[1]}\left(w_{1}\right) W^{[2]}\left(w_{2}\right) .
$$

Plugging this into (6.1), we find that

$$
\begin{gathered}
-\frac{\hbar^{2}}{2 \mu}\left\{\frac{1}{r_{1}^{2}} \frac{\partial}{\partial r_{1}}\left(r_{1}^{2} \frac{\partial R}{\partial r_{1}}\right)+\frac{1}{r_{2}^{2}} \frac{\partial}{\partial r_{2}}\left(r_{2}^{2} \frac{\partial R}{\partial r_{2}}\right)-\left(\frac{\ell_{1}\left(\ell_{1}+1\right)}{r_{1}^{2}}+\frac{\ell_{2}\left(\ell_{2}+1\right)}{r_{2}^{2}}\right) R\right\} \\
-\left\{Z e^{2}\left(\frac{1}{r_{1}}+\frac{1}{r_{2}}\right)+\left(\left(\Gamma_{\mathcal{M}}\right)_{k_{1}}+\left(\Gamma_{\mathcal{M}}\right)_{k_{2}}+E\right)\right\} R+\frac{e^{2}}{\left|r_{1}-r_{2}\right|} R=0, \\
\frac{1}{\sin \left(\theta_{i}\right)} \frac{\partial}{\partial \theta_{i}}\left(\sin \left(\theta_{i}\right) \frac{\partial Y^{[i]}}{\partial \theta_{i}}\right)+\frac{1}{\sin ^{2}\left(\theta_{i}\right)} \frac{\partial^{2} Y^{[i]}}{\partial \phi_{i}^{2}}+\ell_{i}\left(\ell_{i}+1\right) Y^{[i]}=0, \quad(i=1,2), \\
-\frac{\hbar^{2}}{2 \mu} \triangle_{\mathcal{M}_{i}} W^{[i]}+\left(\Gamma_{\mathcal{M}}\right)_{k_{i}} W^{[i]}=0, \quad(i=1,2) .
\end{gathered}
$$

From here, much of the analysis is similar to that presented in Sec. II for the Hydrogen-like atom, and we do not repeat the elementary steps. The primary difference here is that the radial equation is a partial differential equation which does not separate (due to the coupling term $1 /\left|r_{1}-r_{2}\right|$ ), and this shall be the focus of our discussion. At this juncture, there are two possibilities: One may either (i) attempt to directly solve the linear PDE (6.5) exactly or (ii) apply a simplifying assumption so that Eq. (6.5) decouples in $r_{1}$ and $r_{2}$. While the PDE (6.5) can be solved numerically for suitable boundary conditions, solving the linear PDE exactly is very difficult due to the coupling term $1 /\left|r_{1}-r_{2}\right|$, which rules out possibility (i) (as we seek to obtain the general spectrum, rather than numerical results for fixed parameters). As we are interested in general, yet approximate order-of-magnitude-type results (recall that we have already made a strong assumption on the form of the potential $U$ ), approximate solutions arising from reasonable assumptions will be acceptable. In the regime where $r_{1} \gg r_{2}$,

$$
\frac{1}{\left|r_{1}-r_{2}\right|}=\frac{1}{r_{1}}\left(\frac{1}{1-\frac{r_{2}}{r_{1}}}\right) \approx \frac{1}{r_{1}},
$$

which permits separation of variables for (6.5). We find that

$$
R\left(r_{1}, r_{2}\right)=\frac{\text { constant }}{r_{1} r_{2}} \mathrm{M}\left(-\frac{G_{1}-G_{3}}{2 \sqrt{\sigma}}, \ell_{1}+\frac{1}{2}, 2 \sqrt{\sigma} r_{1}\right) \mathrm{M}\left(-\frac{G_{1}}{\sqrt{G_{2}-\sigma}}, \ell_{2}+\frac{1}{2}, 2 \sqrt{G_{2}-\sigma} r_{2}\right),
$$


where the constants $G_{i}$ are defined as

$$
G_{1}=Z G_{3}=-\frac{2 \mu Z e^{2}}{\hbar^{2}}, \quad G_{2}=-\frac{2 \mu}{\hbar^{2}}\left(\left(\Gamma_{\mathcal{M}}\right)_{k_{1}}+\left(\Gamma_{\mathcal{M}}\right)_{k_{2}}+E\right)
$$

while $\sigma$ represents the spectrum of constants due to separation of variables. The constants $\sigma$ may be determined by the regularity of the first factor at the origin, and we obtain the constants

$$
\sigma_{N_{1}}=\frac{(Z-1)^{2} e^{4}}{4\left(N_{1}+1\right)^{2}}\left(\frac{2 \mu}{\hbar^{2}}\right)
$$

Then, due to the desired regularity of the second factor at the origin, the energy spectrum will take the form

$$
E_{N_{1}, N_{2}, k_{1}, k_{2}}=-E_{N_{1}, N_{2}}^{*}-\left(\Gamma_{\mathcal{M}}\right)_{k_{1}}-\left(\Gamma_{\mathcal{M}}\right)_{k_{2}}, \quad E_{N_{1}, N_{2}}^{*}=\frac{\mu e^{4}}{2 \hbar^{2}}\left(\frac{(Z-1)^{2}}{\left(N_{1}+1\right)^{2}}+\frac{Z^{2}}{\left(N_{2}+1\right)}\right) .
$$

Note that (6.5) is symmetric (relabelling the $r_{i}$ 's effects nothing), so in the $r_{2} \gg r_{1}$ regime we swap $r_{1}$ and $r_{2}$ in 6.8 .

Such results as (6.8) and (6.9) capture the asymptotic behavior of the solutions, and so we are then left to consider the regimes in which $r_{1}$ and $r_{2}$ are sufficiently close. Motivated by the $\delta$-expansion method of Bender et al., ${ }^{25}$ let us replace the coupling term $1 /\left|r_{1}-r_{2}\right|$ with $1 /\left|r_{1}-r_{2}\right|^{\delta}$. We shall construct a perturbation solution in $\delta$ of the form

$$
R\left(r_{1}, r_{2} ; \delta\right)=R_{0}\left(r_{1}, r_{2}\right)+R_{1}\left(r_{1}, r_{2}\right) \delta+O\left(\delta^{2}\right),
$$

which gives the order zero and one equations as

$$
\begin{gathered}
-\frac{\hbar^{2}}{2 \mu}\left\{\frac{1}{r_{1}^{2}} \frac{\partial}{\partial r_{1}}\left(r_{1}^{2} \frac{\partial R_{0}}{\partial r_{1}}\right)+\frac{1}{r_{2}^{2}} \frac{\partial}{\partial r_{2}}\left(r_{2}^{2} \frac{\partial R_{0}}{\partial r_{2}}\right)-\left(\frac{\ell_{1}\left(\ell_{1}+1\right)}{r_{1}^{2}}+\frac{\ell_{2}\left(\ell_{2}+1\right)}{r_{2}^{2}}\right) R_{0}\right\} \\
(6.5)-\left\{Z e^{2}\left(\frac{1}{r_{1}}+\frac{1}{r_{2}}\right)+\left(\left(\Gamma_{\mathcal{M}}\right)_{k_{1}}+\left(\Gamma_{\mathcal{M}}\right)_{k_{2}}+E\right)\right\} R_{0}+e^{2} R_{0}=0,
\end{gathered}
$$

and

$$
\begin{aligned}
& -\frac{\hbar^{2}}{2 \mu}\left\{\frac{1}{r_{1}^{2}} \frac{\partial}{\partial r_{1}}\left(r_{1}^{2} \frac{\partial R_{1}}{\partial r_{1}}\right)+\frac{1}{r_{2}^{2}} \frac{\partial}{\partial r_{2}}\left(r_{2}^{2} \frac{\partial R_{1}}{\partial r_{2}}\right)-\left(\frac{\ell_{1}\left(\ell_{1}+1\right)}{r_{1}^{2}}+\frac{\ell_{2}\left(\ell_{2}+1\right)}{r_{2}^{2}}\right) R_{1}\right\} \\
& (6.5)-\left\{Z e^{2}\left(\frac{1}{r_{1}}+\frac{1}{r_{2}}\right)+\left(\left(\Gamma_{\mathcal{M}}\right)_{k_{1}}+\left(\Gamma_{\mathcal{M}}\right)_{k_{2}}+E\right)\right\} R_{1}+e^{2} R_{1}=\ln \left|r_{1}-r_{2}\right| R_{0},
\end{aligned}
$$

respectively, where we have used the fact

$$
\frac{1}{\left|r_{1}-r_{2}\right|^{\delta}}=1-\ln \left|r_{1}-r_{2}\right| \delta+\frac{1}{2}\left(\ln \left|r_{1}-r_{2}\right|\right)^{2} \delta^{2}+O\left(\delta^{3}\right) .
$$

The latter of the two equations highlights the fact that we have a logarithmic singularity as $r_{1} \rightarrow r_{2}$. Note that the equation governing $R_{0}$ is separable in $r_{1}$ and $r_{2}$, and we obtain $R_{0}\left(r_{1}, r_{2}\right)$ $=R_{0}^{[1]}\left(r_{1}\right) R_{0}^{[2]}\left(r_{2}\right)$. Meanwhile, the equation for $R_{1}$ is not separable, due to the appearance of the inhomogeneous term containing the factor $\ln \left|r_{1}-r_{2}\right|$. One can obtain a Fourier-serieslike expression for $R_{1}$, with base functions of the type Whittaker $M$ and $W$, with summation of the basis functions of index $N_{1}, N_{2}, k_{1}$, and $k_{2}$. Such an expression is complicated to even write, and we omit the details of this. As it turns out, the order zero perturbation will provide the order-of-magnitude-type estimates we desire (far enough away from $r_{1}=r_{2}$ ). For this perturbation expansion, we see that the energy states $E_{N_{1}, N_{2}, k_{1}, k_{2}}$ will still take the form (6.9), the only difference will be in the explicit value of $\bar{E}_{N_{1}, N_{2}}$ (the contribution from $\mathbb{R}^{3}$ ). Thus, in summary, the shift in energy states for the Helium atom which occur due to the assumption of additional small dimensions will be similar in nature to those which occurs for the hydrogen atom as demonstrated earlier in this paper. 


\section{CONCLUSIONS}

We have given consideration to a hydrogenic atom over a space of the form $\mathbb{R}^{3} \times \mathcal{M}$, where $\mathcal{M}$ may be a generalized manifold obeying certain properties. Upon separating the solution to the governing time-independent Schrödinger equation into a component over $\mathbb{R}^{3}$ and a component over $\mathcal{M}$, we are able to recover both the wave functions and energy spectrum for the hydrogenic atom over $\mathbb{R}^{3} \times \mathcal{M}$ in a general setting. We proceed to discuss why the case in which the underlying space is of the form $\mathbb{R}^{D}$ for $D \geq 4$ is not physically reasonable. We then consider an example of a relatively simple manifold structure by setting $\mathcal{M}=T^{D}$, the $D$-dimensional torus. From here, we set $\mathcal{M}=\mathcal{K}$, where $\mathcal{K}$ denotes a Kähler conifold. We present the basis of wave functions, along with the resulting energy spectrum, for the hydrogenic atom in each case. It is found that the assumption of compact extra dimensions results in additive corrections to the standard energy spectrum for the hydrogen atom over $\mathbb{R}^{3}$. Furthermore, such corrections are expected to be quite large, as the corrections scale as $1 / L^{2}$, where $L$ denotes the size of the additional dimensions. Thus, under the assumption of relatively small compact extra dimensions, even the first corrections accounting for these extra dimensions to the standard energy spectrum will be quite large.

This area appears ripe for future research. We consider one fairly simple example, along with a more realistic (and, hence, complicated) example for $\mathcal{M}$. In the future, the corresponding results for more exotic generalized manifolds $\mathcal{M}$ might be considered. Indeed, for any such compact structures, we expect the same general behavior of the energy spectrum: That is to say, we expect that any corrections to the standard energy spectrum for hydrogen will be of relatively large magnitude, assuming that the extra dimensions (if any) are small. Furthermore, one might consider extending the present results to other atoms. While such endeavors should prove computationally challenging, we posit that it should be quite possible to obtain the energy spectrum for such atoms, at least numerically, for a number of specific choices for $\mathcal{M}$.

\section{ACKNOWLEDGMENTS}

An earlier manuscript, on which part of this work was based, was supported in part by NSF Grants DMS0649159 and DMS0508779. The author thanks C. J. Efthimiou for his suggestion of a similar problem, and for reading and earlier form of the present manuscript and offering his valuable comments. The authors also thanks T. Hübsch for reading an earlier copy of the manuscript, and correcting some errors in the presentation.

${ }^{1}$ G. Nordström, "Über die Möglichkeit, das elektromagnetische Feld und das Gravitationsfeld zu vereinigen," Phys. Zeitschrift 15, 504 (1914).

${ }^{2}$ T. Kaluza, "Zum Unitätsproblem in der Physik, Sitzungsber,” Preuss. Akad. Wiss. Berlin. (Math. Phys.) 966 (1921). O. Klein, "Quantentheorie und fünfdimensionale Relativitätstheorie," Z. Phys. 37, 895 (1926); E. Witten, "Search for a realistic Kaluza-Klein theory,” Nucl. Phys. B 186, 412 (1981).

${ }^{3}$ I. Mladenov and V. Tsanov, "Geometric quantization of the multidimensional Kepler problem," J. Geom. Phys. 2, 17 (1985).

${ }^{4}$ E. T. Whittaker, “An expression of certain known functions as generalised hypergeometric functions," Bull. Amer. Math. Soc. 10, 125 (1904); C. S. Meijer, "Über die Integraldarstellungen der Whittakerschen Funktion $W_{(k, m)}(z)$ und der Hankelschen und Besselschen Funktionen,” Nieuw Arch. Wisk. 18, 35 (1936); M. Abramowitz and I. A. Stegun, (Eds.). "Confluent hypergeometric functions." in Handbook of Mathematical Functions with Formulas, Graphs, and Mathematical Tables, 9th ed. (Dover, New York, 1972), pp. 503-515; S. Iyanaga and Y. Kawada, (Eds.). "Whittaker functions," Appendix A, Table 19.II in Encyclopedic Dictionary of Mathematics (MIT, Cambridge, MA, 1980), pp. 1469-1471.

${ }^{5}$ V. Aquilanti, S. Cavalli, and C. Coletti, "The D-dimensional hydrogen atom: hyperspherical harmonics as momentum space orbitals and alternative Sturmian basis sets," Chem. Phys. 214, 1 (1997); H. Hosoya, "Hierarchical structure of the atomic orbital wave functions of D-dimensional atom," J. Phys. Chem. A 101, 418 (1997); H. Hosoya, "Pascal's triangle, non-adjacent numbers, and D-dimensional atomic orbitals," J. Mat. Chem. 23, 169 (1998); S. Ka-Lin and L. An-ling, "D-dimensional q-harmonic oscillator and d-dimension q-hydrogen atom," Int. J. Theor. Phys. 38, 2289 (1999); A. Wipf, A. Kirchberg, and D. Länge, "Algebraic solution of the supersymmetric hydrogen atom,” Bulg. J. Phys. 33206 (2006); G.-J. Zeng, K.-L. Su, and M. Li, "Most general and simplest algebraic relationship between energy eigenstates of a hydrogen atom and a harmonic oscillator of arbitrary dimensions," Phys. Rev. A 50, 4373 (1994).

${ }^{6} \mathrm{H}$. Hosoya, "Back-of-envelope derivation of the analytical formulas of the atomic wave functions of a D-dimensional atom," Int. J. Quantum Chem. 64, 35 (1997); J. C. Carzoli, M. Dunn, and D. K. Watson, "Singly and doubly excited states of the D-dimensional helium atom," Phys. Rev. A 59, 182 (1999).

${ }^{7}$ C. J. Efthimiou and D. A. Spector, "A collection of exercises in two-dimensional physics, part 1," e-print arXiv:hepth/0003190v1 (2000); S. Nouri, "Generalized coherent states for the d-dimensional Coulomb problem," Phys. Rev. A 60, 1702 (1999). 
${ }^{8}$ F. J. Asturias and S. R. Aragon, "The hydrogenic atom and the periodic table of the elements in two spatial dimensions," Am. J. Phys. 53, 893 (1985); T. Negadi and M. Kibler, "The periodic table in flatland," Int. J. Quantum Chem. 57, 53 (1996).

${ }^{9}$ K. Andrew and J. Supplee, "A hydrogenic atom in d-dimensions," Amer. J. Phys. 58, 1177 (1990); P. Ehrenfest, "In what way does it become manifest in the fundamental laws of physics that space has three dimensions?," Proc. Amsterdam Acad. 20, 200 (1917); M. M. Nieto, "Existence of bound states in continuous $0<D<\infty$ dimensions, Phys. Lett. A 293, 10 (2002).

${ }^{10}$ F. Burgbachera, C. Lämmerzahlb, and A. Maciasc, "Is there a stable hydrogen atom in higher dimensions?,” J. Math. Phys. 40, 625 (1999).

${ }^{11}$ K. Greisen, "End to the cosmic-ray spectrum?," Phys. Rev. Lett. 16, 748 (1966); G. T. Zatsepin and V. A. Kuzmin, "Upper limit of the spectrum of cosmic rays," J. Exp. Theor. Phys. Lett. 4, 78 (1966); R. U. Abbasi, et al., "First observation of the Greisen-Zatsepin-Kuzmin suppression," Phys. Rev. Lett. 100, 101 (2008).

${ }^{12}$ W. V. D. Hodge, "Differential forms on a Kähler manifold," Proc. Cambridge Phil. Soc. 47, 504 (1951); P. R. Garabedian and D. C. Spencer, "A complex tensor calculus for Kähler manifolds," Acta Math. 89, 279 (1953); E. Calabi and E. Vesentini, "On compact, locally symmetric Kähler manifolds," Ann. Math. 71, 472 (1960); P. Deligne, P. Griffiths, J. Morgan, and D. Sullivan, "Real homotopy theory of Kähler manifolds," Invent. Math. 29, 245 (1975); A. Gray, "The structure of nearly Kähler manifolds," Math. Annal. 223, 233 (1976); I. Vaisman, "On locally and globally conformal Kähler manifolds," Trans. Amer. Math. Soc. 262, 533 (1980); Y. T. Siu and S. T. Yau, "Compact kähler manifolds of positive bisectional curvature," Invent. Math. 59, 189 (1980); L. A. Cordero, M. Fernandez and M. de Leon, "Examples of compact Non-Kähler almost Kähler manifolds," Proc. Amer. Math. Soc. 95, 280 (1985).

${ }^{13}$ B. Zumino, "Supersymmetry and Kähler manifolds," Phys. Lett. B 87, 203 (1979); S. Salamon, "Quaternionic Kähler manifolds," Invent. Math. 67, 143 (1982); D. S. Freed, "Special Kähler manifolds," Commun. Math. Phys. 203, 31 (1999); S. Bellucci and A. Nersessian, "Note on $N=4$ supersymmetric mechanics on Kähler manifolds," Phys. Rev. D 64, 021702 (2001).

${ }^{14}$ E. Calabi, "The space of Kähler metrics," Proc. Internat. Congress Math. Amsterdam, 206 (1954); E. Calabi, “On Kähler manifolds with vanishing canonical class," Algebraic Geometry and Topology. A symposium in honor of Solomon Lefschetz (Princeton University Press, Princeton, NJ, 1957), pp. 78-89; S. T. Yau, "On the Ricci curvature of a compact Kähler manifold and the complex Monge-Ampére equation. I," Commun. Pure Appl. Math. 31, 339 (1978) 411; N. Hitchingel, "Generalized Calabi-Yau manifolds," Quart. J. Math. 54, 281 (2003).

${ }^{15}$ S. Bellucci, A. Nersessian and A. Yeranyan, "Quantum mechanics model on a Kähler conifold," Phys. Rev. D 70, 045006 (2004).

${ }^{16}$ A. Nersessian and A. Yeranyan, "Three-dimensional oscillator and Coulomb systems reduced from Kähler spaces," J. Phys. A: Math. Gen. 37, 2791 (2004).

${ }^{17}$ S. Bellucci, A. Nersessian and A. Yeranyan, "Quantum oscillator on $\mathbb{C} P^{n}$ in a constant magnetic field," Phys. Rev. D 70, 085013 (2004).

${ }^{18}$ E. Wigner, "On the quantum correction for thermodynamic equilibrium," Phys. Rev. 40, 749 (1932); A Royer, "Measurement of the Wigner function," Phys. Rev. Lett. 55, 2745 (1985); W. K. Wootters, "A Wigner-function formulation of finite-state quantum mechanics," Ann. Phys. 176, 1 (1987); C. R. Leavens and R. Sala Mayato, "On constructing the wave function of a quantum particle from its Wigner phase-space distribution function," Phys. Lett. A 280, 163 (2001); Q. S. Li, G. M. Wei, and L. Q. Lü, "Relationship between the Wigner function and the probability density function in quantum phase space representation," Phys. Rev. A 70, 022105 (2004); N. Costa Dias and J. N. Prata, "Deformation quantization and Wigner functions," Mod. Phys. Lett. A 20, 1371 (2005).

${ }^{19}$ B. R. Holstein, "Quantum mechanics in momentum space: The Coulomb system," Amer. J. Phys. 63, 710 (1995); S. Nouri, "Wigner phase-space distribution function for the hydrogen atom," Phys. Rev. A 57, 1526 (1998); L. Praxmeyer, J. Mostowski, and K. Wódkiewicz, "Hydrogen atom in phase space: the Wigner representation," J. Phys. A: Math. Gen. 39, 14143 (2006).

${ }^{20}$ C. F. Gauss, "Disquisitiones Generales Circa Seriem Infinitam $[(\alpha \beta) /(1 \cdot \gamma)] x+[(\alpha(\alpha+1) \beta(\beta+1)) /(12 \gamma(\gamma+1))] x^{2}+$ $[(\alpha(\alpha+1)(\alpha+2) \beta(\beta+1)(\beta+2)) /(123 \gamma(\gamma+1)(\gamma+2))] x^{3}+$ etc. Pars Prior," Commentationes Societiones Regiae Scientiarum Gottingensis Recentiores, Vol. II. 1812. Reprinted in Gesammelte Werke, Bd. 3, pp. 123-163 and 207-229, 1866; F. Beukers, "Gauss' hypergeometric function," Progress in Mathematics (Birkhäuser Verlag AG, Basel, Switzerland, 2007), Vol. 260, pp. 23-42.

${ }^{21}$ M. Berger, P. Gauduchon and E. Mazet, Le Spectre d'une Variete Riemannienne, Lecture Notes in Mathematics, Vol. 194 (Springer-Verlag, Berlin, 1971).

${ }^{22} \mathrm{P}$. Li and S. T. Yau. "Estimate of eigenvalues of a compact Riemannian manifold." Proceedings of the Symposium in Pure Mathematics (American Mathematical Society, Providence, RI, 1980), Vol. 36, pp. 205-235.

${ }^{23}$ H. C. Yang and J. Q. Zhong, "On the estimate of the first eigenvalue of a compact Riemannian manifold," Scientia Sinica Series A 27, 1265 (1984).

${ }^{24}$ D. Yang, "Lower bound estimates of the first eigenvalue for compact manifolds with positive Ricci curvature," Pacific J. Math. 190, 383 (1999).

${ }^{25}$ C. M. Bender, K. A. Milton, M. Moshe, S. S. Pinsky, and L. M. Simmons, Jr., "Novel perturbative scheme in quantum field theory," Phys. Rev. D 37, 1472 (1988); C. M. Bender, K. A. Milton, S. S. Pinsky, and L. M. Simmons, Jr., "A new perturbative approximation applied to supersymmetric quantum field theory," Phys. Lett. B 205, 493 (1988); C. M. Bender and H. F. Jones, "New nonperturbative calculation: Renormalization and the triviality of $\left(\lambda \phi^{4}\right)_{4}$ field theory," Phys. Rev. D 38, 2526 (1988); C. M. Bender and K. A. Milton, "New perturbative calculation of the Fermion-Boson mass ratio in a supersymmetric quantum field theory," Phys. Rev. D 38, 1310 (1988). 